\title{
Comparação entre a intensidade do esforço realizada por jovens futebolistas no primeiro e no segundo tempo do jogo de Futebol
}

\author{
Lucas Mortimer \\ Luciano Condessa \\ Vinícius Rodrigues \\ Daniel Coelho \\ Danusa Soares \\ Emerson Silami-Garcia
}

\author{
Universidade Federal de Minas Gerais \\ Escola de Educação Física, Fisioterapia e Terapia Ocupacional \\ Centro de Excelência Esportiva (CENESP) \\ Belo Horizonte \\ Minas Gerais \\ Brasil
}

\section{RESUMO}

Constitui objetivo deste estudo comparar a intensidade do esforço (IE) de atletas de futebol entre o primeiro e o segundo tempo de jogos oficiais. Métodos: Foram avaliados 25 atletas (17,5 $\pm 1,2$ anos; $8,5 \pm 1,0 \%$ de gordura corporal; $175,1 \pm 6,8$ $\mathrm{cm} ; 69,3 \pm 5,2 \mathrm{~kg}$ e $\mathrm{VO}_{2 \text { máx }}$ de $\left.52,2 \pm 3,3 \mathrm{mlO}_{2} \bullet \mathrm{kg}^{-1} \bullet \mathrm{min}^{-1}\right)$ pertencentes a um clube da primeira divisão do futebol brasileiro. A freqüência cardíaca (FC) dos atletas foi medida usando-se um conjunto de cardiofrequencímetros, durante jogos de competições oficiais, sendo 14 jogos da categoria juvenil (menos de 17 anos) e 8 jogos da categoria júnior (menos de 21 anos). A freqüência cardíaca máxima $\left(\mathrm{FC}_{\text {máx }}\right)$ de cada atleta foi considerada como o maior valor de FC observado entre dois testes máximos (corrida de $1000 \mathrm{~m}$ e $2400 \mathrm{~m}$ ) utilizados para a determinação da mesma. Nos casos em que foram observados valores superiores da FC durante as situações de jogo em relação aos testes, esta foi considerada como a $\mathrm{FC}_{\text {máx }}$ individual. Os resultados foram analisados usando-se o teste t-Student com nível de significância adotado de $\mathrm{p}<0,05$. Resultados: Foi observada diferença $(\mathrm{p}<0,01)$ da FC média e do percentual da freqüência cardíaca máxima $\left(\% \mathrm{FC}_{\text {máx }}\right)$ entre o primeiro tempo $\left(170 \pm 8\right.$ bpm e $85,2 \pm 4,5 \% \mathrm{FC}_{\text {máx }}$ ) e o segundo tempo (166 \pm $10 \mathrm{bpm}$ e $\left.82,7 \pm 4,6 \% \mathrm{FC}_{\text {máx }}\right)$. Os resultados traduzem uma menor intensidade do esforço no segundo tempo de jogo, comparativamente com o primeiro tempo.

Palavras-chave: futebol, freqüência cardíaca, intensidade de esforço.

\section{ABSTRACT \\ Comparison between the effort intensity of young soccer players in the first and second halves of the soccer game}

Objective: Compare the effort intensity (IE) of young soccer players between the first and second halves of official games. Methods: 25 athletes were evaluated $(17,5 \pm 1,2$ years; $8,5 \pm 1,0 \%$ of body fat; $175,1 \pm 6,8 \mathrm{~cm} ; 69,3 \pm 5,2 \mathrm{~kg}$ and $\mathrm{VO}_{2 \text { máx }}$ of $52,2 \pm 3,3 \mathrm{mlO}_{2} \cdot$ $\mathrm{kg}^{-1} \bullet \mathrm{min}^{-1}$ ) who pertained to a club of the first division of the Brazilian soccer. Heart rate (HR) of the athletes was measured using a set of heart rate monitors, during official games, being 14 games of the under-17 category (U-17) and 8 games of the under-20 category (U20). The maximal heart rate (MHR) for each athlete was considered as the highest HR observed during two maximal effort tests (race of $1000 \mathrm{~m}$ and $2400 \mathrm{~m}$ ). In the cases which a higher value of HR was observed during game situations in relation to the tests, this was considered the MHR. Results were analyzed using Student $t$ test and the accepted level of significance was $p<0,05$. Results: There was a significant difference $(p<0,01)$ between the average $H R$ and the percentage of maximal heart rate (\%MHR) between first half $(170 \pm 8 \mathrm{bpm}$ and $85,2 \pm 4,5 \% \mathrm{MHR})$ and second half (166 $\pm 10 \mathrm{bpm}$ e 82,7 \pm $4,6 \% M H R)$. These results show a lower effort intensity in the second half of the game, in comparison with the first half.

Key Words: soccer, heart rate, effort intensity. 


\section{INTRODUÇÃO}

O futebol é considerado como um dos esportes mais praticados no mundo (15), existindo, atualmente, mais de duzentos milhões de jogadores em atividade (12). Desta forma, vem crescendo o interesse dos pesquisadores da área da educação física sobre a demanda fisiológica durante a prática deste esporte. Uma maior compreensão deste tema pode permitir um aperfeiçoamento dos programas de treinamentos, o que levaria os jogadores a obterem um melhor desempenho durante os jogos (32).

A demanda fisiológica à qual um indivíduo está submetido durante uma partida de futebol tem sido relatada a partir de diferentes parâmetros, como a distância total percorrida $(5,6,10,15,23,26)$, a velocidade média de corrida (5), a temperatura corporal $(3,10)$, medidas diretas de oxigênio $(21)$, concentração de lactato $(5,10)$ e freqüência cardíaca (FC) $(2,3,5,8,9,10,13,15,20,21,25,26)$.

Dentre esses parâmetros, a determinação da intensidade do esforço (IE) através da medição direta do consumo de oxigênio $\left(\mathrm{VO}_{2}\right)$ dos jogadores durante o jogo seria uma fonte de informação bastante esclarecedora. Porém, é clara a interferência que a utilização dos instrumentos que este método exige pode acarretar para o rendimento dos atletas durante o jogo. Além disso, existe ainda a proibição do uso de quaisquer equipamentos pelos jogadores em jogos oficiais (26). Devido a esta limitação, somente jogos simulados poderiam ser avaliados. No estudo de Reilly (1997) somente uma pequena parte do jogo simulado pôde ser avaliada, sendo estes resultados extrapolados para todo o jogo, o que não representa a verdadeira IE do mesmo.

Portanto, dentre todos os parâmetros utilizados para a determinação da IE no futebol, a FC apresenta-se como uma variável de fácil aplicação para a determinação da produção de energia aeróbica $(1,11,13$, $14,29,31)$ durante um jogo de futebol $(2,8)$. Isto se deve ao fato da existência de uma relação linear entre a $\mathrm{FC}$ e o $\mathrm{VO}_{2}(5,11)$, mesmo em exercícios intermitentes como o futebol (5).

Considerando que a FC sofre interferência de alguns fatores, como por exemplo, a idade, Karvonen e Vuorimaa (1988) recomendam a sua relativização em função da freqüência cardíaca máxima $\left(\mathrm{FC}_{\text {máx }}\right)$. Assim, a IE indicada como percentual da freqüência cardíaca máxima $\left(\% \mathrm{FC}_{\text {máx }}\right)$ representa um parâmetro adequado para o controle da IE de atletas e praticantes recreacionais de atividades físicas (16).

Pela alta intensidade e longa duração de um jogo de futebol, os jogadores devem ser capazes de manter um alto nível de esforço durante todo o jogo. No entanto, identifica-se um declínio na distância percorrida, na intensidade de trabalho, na FC, nas concentrações de lactato e de glicose no decorrer do jogo $(28,33)$, apontando para uma menor permanência dos jogadores em zonas de maior IE no decorrer do jogo (15). Em geral, a distância percorrida por um jogador durante um jogo de futebol depende do grau de dificuldade imposto pelo time adversário, de aspectos táticos adotados pelas equipes e da importância do jogo (7). O mesmo autor identificou ainda a existência de diferença entre a distância percorrida por uma equipe entre o primeiro e segundo tempo de jogo, sendo a distância percorrida no segundo tempo menor em cerca de $5 \%$. Vários outros autores $(2,5,8,15,19)$ também encontraram diferenças entre a IE no primeiro e segundo tempos, utilizando a FC como parâmetro de avaliação da mesma.

Tem sido sugerido por alguns autores $(28,33)$ que os jogadores podem apresentar uma melhora no desempenho técnico e tático durante os jogos se melhorarem sua capacidade aeróbia. Considerando que os jogadores de futebol que possuem alta capacidade aeróbia são capazes de percorrer maiores distâncias durante um jogo (30), participar de um número maior de jogadas decisivas, aumentar o número de sprints realizados (15), melhorar a sua recuperação após os sprints (4), atenuar a diminuição do desempenho no segundo tempo de jogo (10) e aumentar o desempenho global durante uma partida de futebol $(28,33)$, sugere-se uma maior ênfase no treinamento da capacidade aeróbia no futebol, visando diminuir a queda da IE dos jogadores ao longo do jogo (15).

Poucos estudos têm sido apresentados na literatura sobre a IE de jogadores de futebol brasileiros. A maioria dos estudos sobre IE no futebol é realizada em países europeus de clima temperado ou frio (28). Tendo em vista que o Brasil possui uma das melhores escolas de futebol do mundo, tendo obtido títulos mundiais nas principais categorias (tetracampeão 
sub-17, tetracampeão sub-20 e pentacampeão profissional), e que o clima da região é considerado tropical, faz-se relevante a análise da IE nesta população. Além disso, o estilo de jogo das equipas brasileiras pode ser considerado diferente daquele adotado pelas equipas européias, o qual poderia influenciar na intensidade de esforço do jogo (23).

Portanto, o objetivo do presente estudo foi comparar a IE, expressa como valores absolutos em batimentos por minuto (bpm) e como $\% \mathrm{FC}_{\text {máx }}$, de futebolistas entre o primeiro e o segundo tempo de jogos oficiais.

\section{MÉTODOS \\ Amostra}

Participaram do estudo 25 atletas do sexo masculino, pertencentes a um clube da primeira divisão do futebol brasileiro, que mantêm treinamentos regulares e participação em competições reconhecidas pela Confederação Brasileira de Futebol (CBF). Os jogadores de futebol treinavam em média 16 horas por semana e jogavam nos campeonatos duas vezes por semana (geralmente, quartas-feiras e domingos). Os jogadores possuíam em média 5 anos de experiência com o futebol com treinamento sistematizado em clubes e, grande parte destes, já faziam parte da categoria de base do clube em questão. As características desses atletas estão descritas no Quadro 1.

Quadro 1. Idade, percentual de gordura [\%G], estatura, massa corporal (MC) e capacidade aeróbia $\left[\mathrm{VO}_{2 \text { máx }}\right.$ ] dos futebolistas que fizeram parte da amostra. Valores apresentados como média e desvio padrão.

\begin{tabular}{|c|c|c|c|c|c|c|c|c|c|c|c|c|c|}
\hline \multirow{2}{*}{$\begin{array}{l}\text { Categoria } \\
\text { Juvenil }\end{array}$} & \multirow{2}{*}{$\begin{array}{c}\mathbf{n} \\
13\end{array}$} & \multicolumn{3}{|c|}{ Idade [anos] } & \multicolumn{3}{|c|}{ Estatura $[\mathrm{cm}]$} & \multicolumn{3}{|c|}{$\% \mathrm{G}$} & \multicolumn{3}{|c|}{ V02 máx $[\mathrm{mL} 02 \cdot \mathrm{kg}-1 \cdot \mathrm{min}-1]$} \\
\hline & & 16,4 & \pm & 0,5 & 175,0 & \pm & 6,8 & 9,3 & \pm & 1,0 & 56,1 & \pm & 2,0 \\
\hline Júnior & 12 & 18,2 & \pm & 0,7 & 178,0 & \pm & 7,3 & 8,5 & \pm & 1,0 & 58,2 & \pm & 2,9 \\
\hline
\end{tabular}

Este estudo foi aprovado pelo Comitê de Ética em Pesquisa (COEP) da Universidade Federal de Minas Gerais (ETIC-476/2004) e respeitou todas as normas estabelecidas pelo Conselho Nacional da Saúde (Res. 196/96) envolvendo pesquisas com seres humanos. Todos os atletas voluntários assinaram um Termo de Consentimento Livre e Esclarecido onde confirmaram estarem cientes dos objetivos e métodos utilizados e da possibilidade de abandonar o estudo a qualquer momento sem a necessidade de justificativa.

\section{Condições ambientais}

O registro das condições ambientais dos locais de realização dos jogos na data de acontecimento dos mesmos foi fornecido pelo departamento de meteorologia do Centro de Desenvolvimento de Tecnologia Nuclear (CDTN) da UFMG.

A temperatura seca foi em média $23,9 \pm 2,65 \mathrm{C}^{\circ}$, a temperatura úmida foi em média $18,9 \pm 2,5 \mathrm{C}^{\circ}$ e a umidade relativa foi de $63,3 \pm 15,85 \%$.

\section{Procedimentos}

A FC dos atletas, em batimentos por minuto (bpm), foi medida durante jogos de competições oficiais, sendo 14 jogos da categoria juvenil (menos de 17 anos) e 8 jogos da categoria júnior (menos de 20 anos). No total, foram feitos 113 registros individuais de FC da categoria juvenil e 66 registros da categoria júnior. Foram avaliados, em média, de 6 a 10 jogadores a cada jogo e foram considerados apenas aqueles que possuíam mais de 3 jogos completos, sendo descartados aqueles que foram substituídos durante o decorrer dos jogos. Para a medida da FC foi utilizado o equipamento Polar Team System ${ }^{\circledR}$. Este conjunto de cardiofreqüêncímetros permite o registro da FC durante uma atividade sem a utilização de um monitor de punho, o que é proibido em jogos pelas regras do futebol por colocar em risco a integridade do atleta, de seus companheiros e adversários. A taxa de amostragem de FC registrada foi de $5 \mathrm{~s}$ em $5 \mathrm{~s}$ 
A FC média dos jogadores foi avaliada em valores absolutos (bpm) e também como $\% \mathrm{FC}_{\text {máx }}$. Foram considerados os dados do primeiro e segundo tempo e do tempo total de jogo.

A $\mathrm{FC}_{\text {máx }}$ foi determinada como o maior valor da $\mathrm{FC}$ registrado dentre as três situações descritas a seguir: 1) Teste de esforço em corrida com velocidade subjetiva máxima: realizado em terreno gramado plano com o uso de chuteiras, em uma distância de $1000 \mathrm{~m}$. Este teste já era realizado no próprio clube com o objetivo de se medir a $\mathrm{FC}_{\text {máx. }}$ Portanto, os jogadores já estavam familiarizados com o mesmo; 2) Teste de esforço para a estimativa do consumo máximo oxigênio $\left(\mathrm{VO}_{2 \text { máx }}\right)$ (17): consistiu em percorrer uma distância pré-determinada em terreno plano no menor tempo possível. No presente estudo, utilizou-se a distância de $2400 \mathrm{~m}$ em um local já conhecido pelos atletas; 3) $\mathrm{FC}_{\text {máx }}$ durante os jogos: esta foi considerada como o maior valor da FC registrado durante os jogos. A maioria dos jogadores atingiu a $\mathrm{FC}_{\text {máx }}$ durante os jogos. $\mathrm{A} \mathrm{FC}_{\text {máx }}$ individual determinada dentre as três situações foi utilizada para relativizar o esforço dos jogadores enquanto $\% \mathrm{FC}_{\text {máx }}$.

\section{Análise estatística}

Os dados são apresentados como média e desviopadrão. Os resultados foram analisados usando-se o teste t-Student para amostras dependentes no software SPSS ${ }^{\circledR}$, versão 10.0 e o nível de significância adotado foi de $\mathrm{p}<0,05$.

\section{RESULTADOS}

Os resultados estão apresentados no Quadro 2.

Quadro 2. FC média (bpm] e \%FC ${ }_{\text {máx }}$ das categorias juvenil e júnior e da média das duas categorias [Média], no primeiro tempo, no segundo tempo e no jogo completo. Valores apresentados como média e desvio-padrão (DP).

\begin{tabular}{|c|c|c|c|c|c|c|c|c|c|c|c|c|c|c|c|c|c|c|}
\hline \multirow{3}{*}{$\begin{array}{l}\text { Escalão } \\
\text { Juvenil }\end{array}$} & \multicolumn{6}{|c|}{ Primeiro Tempo } & \multicolumn{6}{|c|}{ Segundo Tempo } & \multicolumn{6}{|c|}{ Jogo Completo } \\
\hline & \multicolumn{3}{|c|}{$\mathrm{FC}$ (bpm) } & \multicolumn{3}{|c|}{ \%FCmáx } & \multicolumn{3}{|c|}{$\mathrm{FC}$ (bpm) } & \multicolumn{3}{|c|}{ \%FCmáx } & \multicolumn{3}{|c|}{$\mathrm{FC}(\mathrm{bpm})$} & \multicolumn{3}{|c|}{ \%FCmáx } \\
\hline & 170 & \pm & 8 & 84,8 & \pm & 4,7 & $165^{*}$ & \pm & 10 & $82,5^{*}$ & \pm & 4,8 & 168 & \pm & 8 & 83,8 & \pm & 4,5 \\
\hline Júnior & 172 & \pm & 10 & 85,9 & \pm & 4,1 & $166^{*}$ & \pm & 10 & $83,0^{*}$ & \pm & 4,4 & 169 & \pm & 9 & 84,5 & \pm & 3,8 \\
\hline Média & 170 & \pm & 8 & 85,2 & \pm & 4,5 & $166^{*}$ & \pm & 10 & $82,7^{*}$ & \pm & 4,6 & 168 & \pm & 8 & 84,1 & \pm & 4,2 \\
\hline
\end{tabular}

*Diferença significativa ( $p<0,01)$ em relação ao primeiro tempo.

Foi observada uma diferença $(\mathrm{p}<0,01)$ da IE, tanto em valores absolutos (bpm) quanto como $\% \mathrm{FC}_{\text {máx }}$, entre o primeiro tempo e o segundo tempo de jogo nas duas categorias. Não houve diferença entre as categorias. A figura 1 apresenta a FC de um jogador ao longo de um jogo oficial. As barras pretas ao longo do eixo tempo demonstram as médias da FC para o primeiro tempo (189 bpm) e segundo tempo (176 bpm).

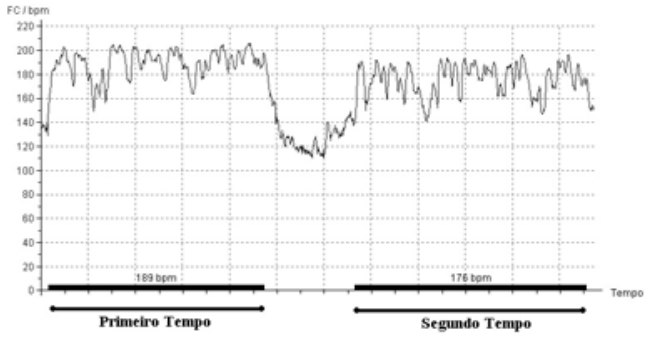

Figura 1. FC de um jogador ao longo de um jogo oficial. 


\section{DISCUSSÃO}

No nosso estudo não houve diferença entre as categorias juvenil e júnior, provavelmente, devido à proximidade da idade, semelhança da carga de treinamento e freqüência de jogos das mesmas. Portanto, a discussão se desenvolverá em torno dos resultados da média das duas categorias.

No segundo tempo de um jogo de futebol a IE realizada pelos atletas diminui, o que pode dever-se à fadiga $(22,23)$. No presente estudo, registrou-se uma queda significativa da IE no segundo tempo $(p<0,01)$ que pode ser visualizada, a título ilustrativo, na figura 1, a qual exibe uma maior FC média de um jogador no primeiro tempo (189 bpm) em comparação ao segundo tempo (176 bpm). Esta queda da IE no segundo tempo de jogo também foi observada em outros estudos $(2,5,19,21,27)$, quer usando como amostra crianças de 11 anos (8), quer quando se analisaram jogos simulados (13).

Possivelmente, este comportamento se deve à degradação progressiva do glicogênio muscular ao longo da partida, o que poderia implicar em uma queda do rendimento físico dos atletas na segunda parte do jogo (5).

A análise da FC de seis atletas de futebol em jogos da liga nacional dinamarquesa mostraram uma redução de 10 bpm na FC média durante o segundo tempo de jogo (154 bpm), comparativamente com a FC média observada no primeiro tempo do jogo (164 bpm) (5). Ali e Farraly (2), comparando jogadores semi-profissionais, universitários e recreacionais, constataram também que os valores médios de FC dos jogadores no segundo tempo eram inferiores aos do primeiro tempo de jogo. Estes resultados corroboram os de Mohr et al. (19), que mostraram valores médios de FC de $164 \pm 1$ e $158 \pm 1 \mathrm{bpm}$ para o primeiro e segundo tempo de jogo, respectivamente. O jogo avaliado neste último estudo citado foi um jogo de futebol amistoso e foi considerada como $\mathrm{FC}_{\text {máx }}$ o maior valor da $\mathrm{FC}$ registrado durante o mesmo. Os resultados do presente estudo corroboram os dos estudos apresentados anteriormente. Em nosso estudo a FC média dos jogadores durante o primeiro tempo $(170 \pm 8 \mathrm{bpm})$ revelou-se superior à do segundo tempo $(166 \pm 10 \mathrm{bpm})$, porém deve-se considerar que a FC média em valores absolutos dos jogadores do presente trabalho mostrou valores mais altos do que os encontrados nos estudos citados anteriormente.

Capranica et al. (8), a partir da avaliação da FC de crianças de 11 anos durante um jogo de futebol, determinou o percentual do tempo de jogo durante o qual estas crianças apresentavam valores da FC superiores a $170 \mathrm{bpm}$. Os autores constataram que as crianças permaneciam $88 \%$ e $80 \%$ do tempo total de jogo, no primeiro e segundo tempo, respectivamente, acima desta FC. Entretanto, os mesmos autores relataram que esses dados não se revelaram estatisticamente diferentes. Convém relevar que, ao contrário do presente estudo, foram avaliadas crianças e a $\mathrm{FC}_{\text {máx }}$ durante o jogo não foi determinada. Nos estudos citados anteriormente a FC média dos jogadores foi considerada em valores absolutos (bpm). No entanto, a relativização da $\mathrm{FC}$ pela $\mathrm{FC}_{\text {máx }}$ revela-se necessária quando se avaliam diferentes indivíduos, já que a $\mathrm{FC}$ e a $\mathrm{FC}_{\text {máx }}$ apresentam variação interindividual (16). No presente estudo a IE realizada pelos jogadores durante o jogo foi em média $85,2 \pm 4,5$ e $82,7 \pm 4,6 \% \mathrm{FC}_{\text {máx }}$ para o primeiro e segundo tempos, respectivamente, revelando-se estes valores significativamente diferentes $(\mathrm{p}<0,01)$. Estudos realizando este tipo de análise (comparação entre o primeiro tempo e o segundo tempo de jogo) não foram encontrados na literatura. No entanto, valores de IE, expressa como $\% \mathrm{FC}_{\text {máx }}$, próximos aos encontrados no nosso estudo para o tempo total do jogo $\left(84,1 \pm 4,2 \% \mathrm{FC}_{\text {máx }}\right)$ foram relatados por outros autores $(18,19,20,21,25)$. O nosso estudo, permite concluir que a intensidade do esforço realizada pelos futebolistas no segundo tempo de jogo, quando comparada com a do primeiro tempo, se revelou inferior, o que sugere uma diminuição do desempenho nesta parte do jogo.

\section{AGRADECIMENTOS}

CNPq, CAPES, FAPEMIG e Ministério do Esporte.

\section{CORRESPONDÊNCIA}

\section{Emerson Silami Garcia}

Rua Rio de Janeiro, 2779, apto. 602,

Lourdes, Belo Horizonte, MG

Brasil

silami@ufmg.br 


\section{REFERÊNCIAS}

1. Achten J, Jeukendrup AE (2003). Heart rate monitoring: applications and limitations. Sports Medicine 33, 7: 517538.

2. Ali A, Farrally M (1991). Recording soccer players' heart rates during matches. Journal of Sports Sciences 9, 183-189.

3. Astrand I, Astrand PO, Christensen EH, Hedman R (1960). Intermittent muscular work. Acta Physiologica Scandinavica 48, 448-453.

4. Aziz AR, Chia M, The KC (2000). The relationship between maximal oxygen uptake and repeated sprint performance indices in field hockey and soccer players. The Journal of Sports Medicine and Physical Fitness 40, 3:195-200.

5. Bangsbo J (1994). The physiology of soccer, with special reference to intense intermittent exercise. Acta Physiologica Scandinavica: an international journal of physiological sciences, v. 151, suplementum 619 .

6. Bangsbo J, Lindquist F (1992). Comparison of various exercise tests with endurance performance during soccer in professional players. International Journal of Sports Medicine 13, 2:125-132.

7. Bangsbo J, Norregaard L, Thorso F (1991). Activity profile of competition soccer. Canadian Journal of Sports Sciences, 16, 2: 110-116.

8. Capranica L, Tessitore A, Guidetti L, Figura F (2001). Heart rate and match analysis in pre-pubescent soccer players. Journal of Sports Sciences 19, 6: 379-384.

9. Drust B, Reilly T, Cable NT (2002). Metabolic and physiological responses to a laboratory-based soccer-specific intermittent protocol on a non-motorised treadmill. In Fourth World Congress of Science and Football, 4, 1999, Sydney. London: E \& FN Spon, 217-225.

10. Ekblom B (1986). Applied Physiology of Soccer. Sports Medicine 3, 50-60.

11. Eston RG, Rowlands AV, Ingledew DK (1998). Validity of heart rate, pedometry, and acceleromety for predicting the energy cost of children's activities. Journal of Applied Physiology 84, 1: 362-371.

12. FIFA - Federation Internationale de Football Association. Disponível em: <www.fifa.com> $>$ Acessado em: Junho de 2005.

13. Flanagan T, Merrick E (2002). Quantifying the work-load of soccer players. In: Fourth World Congress of Science and Football, 4, 1999, Sydney. London: E \& FN Spon, 341-349.

14. Gleim GW, Witmant PA, Nicolas JA (1981). Indirect assessment of cardiovascular "demands" using telemetry on professional football players. The American Journal of Sports Medicine 9, 3: 178-183.

15. Helgerud J, Engen LC, Wisloff U, Hoff J (2001). Aerobic endurance training improves soccer performance. Medicine and Science in Sports Exercise 33, 11: 1925-1931.

16. Karvonen J, Vuorimaa T (1988). Heart hate and exercise intensity during sports activities; practical application. Sports Medicine 5: 303-312.

17. Margaria R, Aghemo P, Pinera LF (1975). A simple relation between performance in running and maximal aerobic power. Journal Applied Physiology 38, 2: 351-352.

18. Miles A, Mclaren D, Reilly T, Yamanaka K (1993). An analysis of physiological strain in four-a-side women's soccer. In Second World Congress of Science and Football, 2, 1991, Eindhoven. Proceedings. London: E \& FN Spon, 140-145.

19. Mohr M, Krustrup P, Nybo L, Nielsen JJ, Bangsbo J
(2004). Muscle temperature and sprint performance during soccer matches - beneficial effect of re-warm-up at half-time. Scandinavian Journal of Medicine and Science in Sports 14, 156-162.

20. O'Connor D (2002). Time-Motion analysis of elite touch players. In Fourth World Congress of Science and Football, 4, 1999, Sydney. London: E \& FN Spon, 126-136.

21. Ogushi T, Ohashi J, Nagahama H, Isokawa S, Suzuki S (1993). Work intensity during soccer match-play (a case study). In Second World Congress of Science and Football, 2, 1991, Eindhoven. Proceedings. London: E \& FN Spon, 121-123.

22. Rebelo, A. N. (2002). Estudo da fadiga no futebol. In Garganta J, Suarez AA, Peñas LC (Eds.) A investigação em futebol: estudos Ibéricos. Porto: Faculdade de Ciências do Desporto e de Educação Física da Universidade do Porto, 121-127.

23. Rienzi E, Drust B, Reilly T, Carter JEL, Martin A (2000). Investigation of antropometric and work-rate profiles of elite south American international soccer player. Journal Sports Medicine and Physical Fitness 40, 162-9.

24. Reilly T (1997). Energetic of high intensity exercise (soccer), with particular reference to fatigue. Journal of Sports Sciences 15: 257-263.

25. Reilly T, Keane S (2002). Estimation of physiological strain on Gaelic football players during match-play. In Fourth World Congress of Science and Football, 4, 1999, Sydney. London: E \& FN Spon, 157-159.

26. Shephard RJ (1992). The energy needs of the soccer player. Clinical Journal of Sports Medicine 2, 1: 62-70.

27. Smith M, Glarke G, Hale T, McMorris T (1993). Blood lactate levels in college soccer players during match-play. In Second World Congress of Science and Football, 2, 1991, Eindhoven. London: E \& FN Spon, 129-134.

28. Stølen T, Chamari K, Castagna C, Wisløff U (2005). Physiology of soccer: an update. Sports Medicine 35, 6: 501536.

29. Strath SJ, AM Swartz, DR Bassett, JR O’Brien WL, GA King, BE Ainsworth (2000). Evaluation of heart rate as a method for assessing moderate intensity physical activity. Medicine and Science in Sports Exercise 32, 9, supplement, S465-S470.

30. Strudwick A, Reilly T, Doran D (2002). Anthropometrics and fitness profiles of elite players in two football codes. Journal of Sports Medicine and Physical Fitness 42, 239-242.

31. Treiber FA, Musante L, Hartdagan S, Davis H, Levy M, Strong WB (1989). Validation of a heart rate monitor with children in laboratory and field settings. Medicine and Science in Sports Exercise 21, 3: 338-342.

32. Wilmore HJ, Haskell WL (1972). Body composition and endurance capacity of professional football players. Journal of Applied Physiology 33, 5: 564-567.

33. Wisloff U, Helgerud J, Hoff J (1998). Strength and Endurance of elite soccer players. Medicine and Science in Sports Exercise 30, 3: 462-467. 